\title{
NUMERICAL INVESTIGATION OF ANISOTROPICALLY DRIVEN DEVELOPED TURBULENCE
}

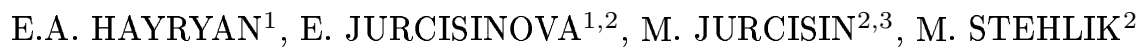 \\ ${ }^{1}$ Laboratory of Information Technologies, Joint Institute for Nuclear \\ Research \\ Joliot-Curie 6, 141980, Dubna, Moscow region, Russia \\ E-mail: ayrjan@jinr.ru; jurcisinova@jinr.ru \\ 2 Institute of Experimental Physics, Slovak Academy of Sciences \\ Watsonova 47, 04001 Košice, Slovakia \\ E-mail: stehlik@saske.sk \\ 3 Laboratory of Theoretical Physics, Joint Institute for Nuclear Research \\ Joliot-Curie 6, 141980, Dubna, Moscow region, Russia \\ E-mail: jurcisin@thsun1.jinr.ru \\ Received October 25, 2006; revised January 16, 2007; published online September 15, 2007
}

\begin{abstract}
The fully developed turbulence with axial anisotropy for dimensions $d>2$ is investigated by means of renormalization group approach. The corresponding system of strongly nonlinear renormalization group equations which contain angle integrals is solved numerically. Possible utilization of the parallel programming methods is discussed. As a result, the influence of anisotropy on the stability of the Kolmogorov scaling regime is analyzed. The borderline dimension between stable scaling regime and unstable one is calculated as a function of the anisotropy parameters. Obtained results are compared with results calculated in [7].
\end{abstract}

Key words: Numerical investigation, developed turbulence, renormalization group

\section{Introduction}

One of the most suitable and also powerful tool for investigation of fully developed turbulence based on the stochastic Navier-Stokes equation [21] is the so-called quantum-field renormalization group (RG) [2, 19]. In early papers, the RG approach was applied only to isotropic models of developed turbulence. However, the method can also be used in the theory of anisotropically developed turbulence. The reason for theoretical study of the influence of 
anisotropy on the behavior of developed turbulence is given by the fact that a variety of experimental studies as well as computer simulations indicate the existence of deviation from the isotropic statistics of the fully developed turbulence. A crucial question immediately arises here: whether the scaling regime remains stable under transition from the isotropic to the anisotropic case. In other words, do the stable fixed points of the RG equations remain stable under the influence of anisotropy?

During the last decade a few papers have appeared in which the above question was considered in the framework of the RG approach in fully developed turbulence and related problems (magnetohydrodynamic developed turbulence, advection of passive and vector fields by a given turbulent environment, etc.). In some cases, it was found that the stability actually takes place (see, e.g., $[13,18])$. On the other hand, the existence of systems without such a stability has also been proven. As was shown in [3] in the anisotropic ${ }^{1}$ magnetohydrodynamic developed turbulence a stable regime generally does not exist. In $[7,13] d$-dimensional models of fully developed turbulence with $d>2$ were investigated for two cases: weak anisotropy [13] and strong anisotropy [7], and it was shown that the stability of the isotropic fixed point is lost for dimensions $d<d_{c} \simeq 2.685$. In [7], where strong anisotropy was investigated, it was also stated that stability of the fixed point, even for dimension $d=3$, takes place only for sufficiently weak anisotropy. In present paper, we would like to return to the problem of the influence of strong anisotropy on the stability of the scaling regime in fully developed turbulence which was studied in [7]. The reason is the suspicion that their results are, at least, not precise (an evidence for this can be also seen by comparison of the results obtained in [7] with results shown in [6]. Thus, in what follows, we shall return to the description and detailed analysis of the numerical methods used in [7]. Our conclusion is the following: the numerical results and conclusions of [7] are not exact and must be specified, although the conceptual framework of their approach is accurate. We shall try to find and to understand the critical points in numerical calculations which could lead to the non accurate results. Another question which will be studied is the possible exploitation of the parallel programming methods to speed up the calculations. As a result, we shall calculate the dependence of the "critical" dimension $d_{c}$ between stable and unstable scaling regimes as a function of the anisotropy parameters and compare our results to that ones obtained in [7].

The paper is organized as follows: In Section 2 we discuss the field theoretic functional formulation of the stochastic problem of fully developed turbulence with strong anisotropy. In Section 3 we discuss the stability of the fixed point under influence of strong anisotropy. In Section 4 we present a detail analysis of numerical methods which was used in calculations. In Section 5 the discussion of the results is present. Appendix I contains explicit expressions of the coefficients of $\beta$ functions. In Appendix II the necessary and sufficient conditions for convergence of needed integrals are proven.

\footnotetext{
${ }^{1}$ In what follows we always have in mind the uniaxial anisotropy, i.e., the anisotropy defined by one specific direction (see the next section).
} 


\section{Description of Model. Field Theoretic Formulation}

In the statistical theory of anisotropically developed turbulence, the turbulent flow is characterized by the random velocity field $\mathbf{v}(\mathbf{x}, t)$, where $\mathbf{v}$ and $\mathbf{x}$ are supposed to be $d$-dimensional vectors. Its evolution is governed by the randomly forced Navier-Stokes equation

$$
\frac{\partial \mathbf{v}}{\partial t}+(\mathbf{v} \cdot \nabla) \mathbf{v}-\nu_{0} \Delta \mathbf{v}-\mathbf{f}^{A}=\mathbf{f},
$$

where the incompressibility of the fluid is assumed, which is given mathematically by the conditions $\nabla \cdot \mathbf{v}=0$ and $\nabla \cdot \mathbf{f}=0$. The parameter $\nu_{0}$ is the kinematic viscosity (subscript 0 denotes bare parameters, see, e.g., [19]). The term $\mathbf{f}^{\mathbf{A}}$ is related to uniaxial anisotropy, and it has the following form [7]

$$
\mathbf{f}^{\mathbf{A}}=\nu_{0}\left[\chi_{10}(\mathbf{n} \nabla)^{2} \mathbf{v}+\chi_{20} \mathbf{n} \nabla^{2}(\mathbf{n v})+\chi_{30} \mathbf{n}(\mathbf{n} \nabla)^{2}(\mathbf{n v})\right] .
$$

Parameters $\chi_{10}, \chi_{20}$ and $\chi_{30}$ characterize the weight of the individual structures in (2.2), and the unit vector $\mathbf{n}$ specifies the direction of the anisotropy axis. The large-scale random force per unit mass $f$ is assumed to have the Gaussian statistics defined by the averages

$$
\left\langle f_{i}\right\rangle=0,\left\langle f_{i}\left(\mathbf{x}_{1}, t\right) f_{j}\left(\mathbf{x}_{2}, t\right)\right\rangle=D_{i j}\left(\mathbf{x}_{1}-\mathbf{x}_{2}, t_{1}-t_{2}\right) .
$$

It is convenient to parametrize the two point correlation matrix

$$
D_{i j}(\mathbf{x}, t)=\delta(t) \int \frac{d^{d} \mathbf{k}}{(2 \pi)^{d}} \tilde{D}_{i j}(\mathbf{k}) \exp (i \mathbf{k} \cdot \mathbf{x})
$$

in the following way $[3,7,18]$

$$
\tilde{D}_{i j}(\mathbf{k})=g_{0} \nu_{0}^{3} k^{4-d-2 \epsilon}\left[\left(1+\alpha_{1} \xi_{k}^{2}\right) P_{i j}(\mathbf{k})+\alpha_{2} R_{i j}(\mathbf{k})\right],
$$

where vector $\mathbf{k}$ is the wave vector, $d$ is the dimension of the space (in our case: $2<d), \epsilon \geq 0$ is dimensionless parameter of the model. The physical value of this parameter is $\epsilon=2$ (so-called energy pumping regime). We shall not discussed here more complicated case $d=2$ (see, e.g., [12] and references cited therein). The value $\epsilon=0$ corresponds to a logarithmic perturbation theory for a calculation of the Green function when $g_{0}$, which plays the role of a bare coupling constant of the model, becomes dimensionless [19]. The problem of the continuation from $\epsilon=0$ to the physical values was discussed in [1]. The $(d \times d)$-matrices $P_{i j}$ and $R_{i j}$ are the transverse projection operators. Their explicit forms are defined by the relations (in the wave-number space)

$$
P_{i j}(\mathbf{k})=\delta_{i j}-\frac{k_{i} k_{j}}{k^{2}}, \quad R_{i j}(\mathbf{k})=\left(n_{i}-\xi_{k} \frac{k_{i}}{k}\right)\left(n_{j}-\xi_{k} \frac{k_{j}}{k}\right),
$$

where $\xi_{k}$ is given by the equation $\xi_{k}=\mathbf{k} \cdot \mathbf{n} / k$. The tensor $\tilde{D}_{i j}$, given by (2.5), is the most general form with respect to the condition of incompressibility of the system under consideration and contains two dimensionless free parameters 
$\alpha_{1}$ and $\alpha_{2}$. The positiveness of the correlator tensor $D_{i j}$ leads to restrictions on the above parameters, namely, $\alpha_{1}>-1$ and $\alpha_{2}>-1$.

Using the well-known Martin-Siggia-Rose formalism [4, 5, 8, 15], the stochastic problem (2.1) with correlator (2.4) can be transformed into the field theoretic model of fields $\mathbf{v}$ and $\mathbf{v}^{\prime}$ where $\mathbf{v}^{\prime}$ is independent of the velocity field $\mathbf{v}$ auxiliary incompressible field, which we have to introduce when transforming the stochastic problem into a functional form. After this transformation the action of the fields $\mathbf{v}$ and $\mathbf{v}^{\prime}$ is given in the form

$$
\begin{aligned}
S & =\frac{1}{2} \int d^{d} \mathbf{x}_{1} d t_{1} d^{d} \mathbf{x}_{2} d t_{2}\left[v_{i}^{\prime}\left(\mathbf{x}_{1}, t_{1}\right) D_{i j}\left(\mathbf{x}_{1}-\mathbf{x}_{2}, t_{1}-t_{2}\right) v_{j}^{\prime}\left(\mathbf{x}_{2}, t_{2}\right)\right] \\
& +\int d^{d} \mathbf{x} d t\left\{\mathbf{v}^{\prime}(\mathbf{x}, t)\left[-\partial_{t} \mathbf{v}-(\mathbf{v} \cdot \nabla) \mathbf{v}+\nu_{0} \nabla^{2} \mathbf{v}+\mathbf{f}^{A}\right](\mathbf{x}, t)\right\} .
\end{aligned}
$$

The functional formulation gives the possibility to use the quantum field theory methods, including the RG technique, to solve the problem. The formulation through action functional (2.7) replaces the statistical averages of random quantities in the stochastic problem (2.1)-(2.5) with equivalent functional averages with weight $\exp \left[S\left(\mathbf{v}, \mathbf{v}^{\prime}\right)\right]$ (for details see $\left.[2,19]\right)$.

\section{RG Equations and Stability of the Kolmogorov Scaling Regime}

Fully developed turbulence is characterized by the large Reynolds number $R e$. On the other hand, the large $R e$ corresponds to the existence of a large inertial interval, which is defined by the inequalities $1 / \Lambda=l \ll r \ll L=1 / m$, where $l$ corresponds to an inner scale (the scale where dissipation forces are dominated, or the scale of the smallest eddies), and $L$ is an outer scale of the system (the scale of the energy pumping into the system, or the scale of the largest eddies). In fully developed turbulence we are interested in the behavior of the correlation functions of velocity field $\left\langle v_{i_{1}}\left(\mathbf{x}_{1}, t\right), \ldots, v_{i_{N}}\left(\mathbf{x}_{N}, t\right)\right\rangle$ deep inside of the inertial interval, i.e., far away from the dissipation effects as well as far away from energy pumping scale. Within the field theoretic approach they are given by the following functional integral (see, [19])

$$
\left\langle v_{i_{1}}\left(\mathbf{x}_{1}, t\right), \cdots, v_{i_{N}}\left(\mathbf{x}_{N}, t\right)\right\rangle=\int \mathcal{D} \Phi v_{i_{1}}\left(\mathbf{x}_{1}, t\right), \cdots, v_{i_{N}}\left(\mathbf{x}_{N}, t\right) e^{S(\Phi)},
$$

where $\Phi=\left\{\mathbf{v}, \mathbf{v}^{\prime}\right\}, 1 \leq i_{j} \leq d, j=1, \ldots, N$, and $S(\Phi)$ is given by $(2.7)$.

The behavior of the correlation functions inside the inertial interval is the main issue of the famous Kolmogorov-Obukhov phenomenological theory $[14,17]$ (see also [16]). It was formulated in the form of two hypotheses which lead to the scaling behavior of the correlation functions within the inertial interval. In what follows we shall discuss only the so-called second Kolmogorov hypothesis related to the IR scaling and our aim will be to investigate the influence of the axial anisotropy on this scaling behavior. 
As was mentioned in the Introduction the appropriate method to investigate self-similar systems is the RG method. Within the RG technique the correlation functions are obtained directly in the scaling form (with correct critical dimensions) and their large-scale limit (i.e., IR limit) is described by the stable fixed points of the renormalization theory, i.e., the scaling regime is stable if the corresponding fixed point is IR stable. The IR fixed point is obtained by using the system of differential equations (also called the flow equations) which drive the effective variables $\bar{C}=\left\{\bar{g}, \bar{\chi}_{1}, \bar{\chi}_{2}, \bar{\chi}_{3}\right\}$ which are the functions of dimensionless scale parameter (wave number) $t=k / \Lambda$. Their explicit form is the following

$$
\left\{\begin{array}{l}
t \frac{d \bar{g}}{d t}=\beta_{g}\left(\bar{g}, \bar{\chi}_{1}, \bar{\chi}_{2}, \bar{\chi}_{3} ; \alpha_{1}, \alpha_{2}, d\right), \\
t \frac{d \bar{\chi}_{i}}{d t}=\beta_{\chi_{i}}\left(\bar{g}, \bar{\chi}_{1}, \bar{\chi}_{2}, \bar{\chi}_{3} ; \alpha_{1}, \alpha_{2}, d\right), \quad i=1,2,3 .
\end{array}\right.
$$

The dimensionless wave number $t$ belongs to the interval $0 \leq t \leq 1$, and the initial conditions for the above differential equations are taken at $t=1$. The IR stable fixed point corresponds to the values in the limit $t \rightarrow 0$, i.e., $\left.\left(\bar{g}, \bar{\chi}_{1}, \bar{\chi}_{2}, \bar{\chi}_{3}\right)\right|_{t \rightarrow 0}=\left(g^{*}, \chi_{1}^{*}, \chi_{2}^{*}, \chi_{3}^{*}\right)$ (standardly, a quantity with star denotes the fixed point value). The so-called $\beta$-functions $\beta_{g}, \beta_{\chi_{i}}, i=1,2,3$ are defined by the so-called renormalization constants of the renormalization procedure and their final form is as follows (details see in Ref. [11])

$$
\beta_{g}=g\left(-2 \epsilon+3 A g a_{1}\right), \quad \beta_{\chi_{i}}=-A g\left(a_{i+1}-\chi_{i} a_{1}\right), \quad i=1,2,3,
$$

where parameter $A$ is defined as $A=S_{d-1} /\left((2 \pi)^{d}\left(d^{2}-1\right)\right), S_{d}$ is the area of the $d$-dimensional sphere given as $S_{d}=2 \pi^{(d / 2)} / \Gamma(d / 2)$, and functions $a_{i}$ $(i=1, \ldots, 4)$ are given in Appendix I.

In principle, one has two possible ways how to find the IR fixed point of the model. First of all, one can solve the system of four equations

$$
\beta_{C}\left(C^{*}, \alpha_{1}, \alpha_{2}, d\right)=0,
$$

where we denote $C^{*}=\left\{g^{*}, \chi_{1}^{*}, \chi_{2}^{*}, \chi_{3}^{*}\right\}$. In this case, the IR stability of the fixed point is determined by the positive real parts of the eigenvalues of the matrix

$$
\omega_{l m}=\left(\frac{\partial \beta_{C_{l}}}{\partial C_{m}}\right)_{C=C^{*}}, \quad l, m=1, \ldots, 4 .
$$

This is a comfortable way for the determination of the fixed point but in our case it cannot be used. The reason is the presence of the integrals in the $\beta$ functions (see Appendix I) which makes this way rather complicated.

The second possibility is to solve directly the system of the differential equations (3.2). It is the way which will be used in our case. This method was also applied in [7]. More about numerical methods will be said in the next section.

Now we have all necessary tools at hand to investigate the fixed point and its stability. Our aim is to find the so-called borderline dimension $d_{c}$ between 

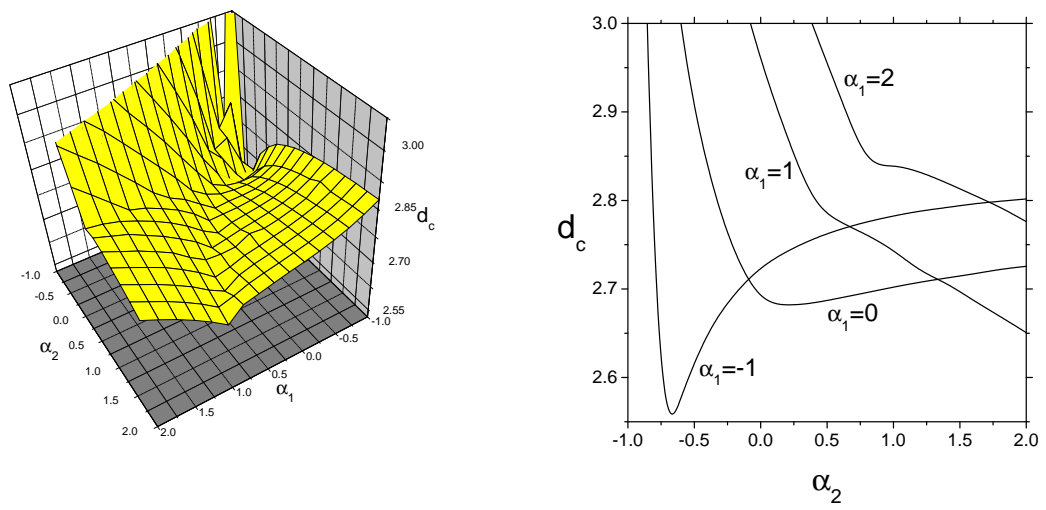

Figure 1. The three-dimensional view on the dependence of the borderline dimension $d_{c}$ on the parameters $\alpha_{1}$, and $\alpha_{2}$.

Figure 2. The dependence of the borderline dimension $d_{c}$ on the parameter $\alpha_{2}$ for concrete values of the parameter $\alpha_{1}$.

stable and unstable regimes when moving from the three-dimensional system towards two-dimensional one. In Fig. 1, and Fig. 2 our results for $d_{c}$ as a function of the anisotropy parameters are present. The difference between our results and those of [7] can be seen immediately, namely, the region of stability of the three-dimensional scaling regime is sufficiently larger in our case than in [7] (see Fig 1 in [7]). Fig. 1, and Fig. 2 show that in the threedimensional system the Kolmogorov scaling regime is unstable only in the limit $\alpha_{1,2} \rightarrow-1$ and for large enough values of parameter $\alpha_{1}$ together with negative or relatively small positive values of the parameter $\alpha_{2}$. Our conclusion is the following (it differs sufficiently from the conclusion found in [7]): to destroy stability of the Kolmogorov scaling regime in three-dimensional space by the uniaxial anisotropy, which is in our model represented by the parameters $\alpha_{1}$, and $\alpha_{2}$, it is necessary to apply anisotropy with rather specific values of these parameters.

\section{Numerical Methods}

One possible way how to solve the problem of the IR fixed point of the four differential RG equations (3.2) with $\beta$ functions (3.3) and corresponding integrals (5.1) of Appendix I is based on the analytical calculations of the integrals. The integrands of (5.1) have the form of fractions of two polynomials, namely: 
$P_{i}\left(x^{2}\right) / Q\left(x^{2}\right)(i=1, \ldots, 4)$, with different numerators $P_{i}\left(x^{2}\right)=b_{i}\left(x^{2}\right)$ but with the same denominator $Q_{i}\left(x^{2}\right)=\left(M_{1} M_{2} M_{3}\right)^{3}$. It is possible to expand the expression $1 /\left(M_{1} M_{2} M_{3}\right)^{3}$ into a sum of partial fractions of the type $R\left(x^{2}\right) /\left(a+x^{2}\right)^{j}$, where $R\left(x^{2}\right)$ is a polynomial, $a$ is, in general, complex function of parameters $\chi_{i}, i=1,2,3$ and $j=1,2,3$. Now using the following result (see, e.g., [9])

$$
\begin{aligned}
\int_{-1}^{1} \frac{\left(1-x^{2}\right)^{\frac{d-3}{2}} x^{2 n}}{\left(a+x^{2}\right)^{j}} d x= & \frac{\left(1+(-1)^{2 n}\right) \Gamma\left(\frac{d-1}{2}\right) \Gamma\left(\frac{1}{2}+n\right)}{2 a^{j} \Gamma\left(\frac{d}{2}+n\right)} \\
& \times{ }_{2} F_{1}\left(1, \frac{1}{2}+n ; \frac{d}{2}+n ;-\frac{1}{a}\right),
\end{aligned}
$$

one can represent the integrals in the form of a combination of hypergeometric functions ${ }_{2} F_{1}(a, b ; c ; z)$ defined as

$$
{ }_{2} F_{1}(a, b ; c ; z)=1+\frac{a b}{1 ! c} z+\frac{a(a+1) b(b+1)}{2 ! c(c+1)} z^{2}+\ldots
$$

Equation (4.1) is held when $\operatorname{Re}[d]>1(\operatorname{Re}[x]$ means the real part of $x)$, $\operatorname{Re}[n]>-1 / 2$, and $\operatorname{Arg}[a] \neq \pi$. In our case, these conditions are fulfilled because $d \in(2,3], n$ is zero or positive integer, and it can be shown that the last condition is also valid.

By using this representation of integrals (5.1) it is possible to find the IR fixed point of differential equations (3.2) by solving the system of equations (3.4) together with the matrix of the first derivatives (3.5) to test the IR stability of the fixed point. But this way is rather complicated and we shall not use it here.

The most comfortable way how to find the IR fixed point of the system of four differential RG equations (3.2) with (3.3) is to solve it numerically using some appropriate numerical method. In what follows, we work with the fourth-order Runge-Kutta method with the adaptive choice of the integration step. For this purpose it is convenient to transform the system of differential equations (3.2) into an autonomic system by substitution $t=e^{-s}$. Using this transformation one obtains

$$
\left\{\begin{array}{l}
\frac{d \bar{g}}{d s}=-\beta_{g}\left(\bar{g}, \bar{\chi}_{1}, \bar{\chi}_{2}, \bar{\chi}_{3} ; \alpha_{1}, \alpha_{2}, d\right), \\
\frac{d \bar{\chi}_{i}}{d s}=-\beta_{\chi_{i}}\left(\bar{g}, \bar{\chi}_{1}, \bar{\chi}_{2}, \bar{\chi}_{3} ; \alpha_{1}, \alpha_{2}, d\right), \quad i=1,2,3,
\end{array}\right.
$$

where $s \in[0, \infty)$. The initial conditions correspond to $s=0$ and the IR fixed point is found in the limit $s \rightarrow \infty$. The first step for the variable $s$ was taken as $\Delta s=10^{-3}$. The initial values of the parameters can be chosen arbitrary but the most convenient choice is to take them to be the fixed point of the three-dimensional isotropic model (see [7]).

As was already discussed our differential equations are made of the linear combinations of the following integrals 


$$
I=\int_{-1}^{1} \frac{\left(1-x^{2}\right)^{\frac{d-3}{2}} x^{2 n}}{\left(M_{1} M_{2} M_{3}\right)^{3}} d x
$$

where explicit forms of the functions $M_{i}, i=1,2,3$ are given in Appendix I. Therefore, the first step to solve the problem is the necessity to guarantee their convergence within the interval $x \in[-1,1]$, i.e., to determine the allowed values of the parameters $\chi_{1}, \chi_{2}, \chi_{3}$. That is why, let us first discuss the conditions under which the integrals will be well defined.

Assume that one or more of the expressions $M_{i},(i=1,2,3)$ (defined in Appendix I) are vanished with respect to the variable $x$ within the interval $[-1,1]$. Let us denote as $x_{1}$ the point in which one of the $M_{i}$ is equal to zero. Then, from the convergence point of view, the integral (4.2) is equivalent to the integral of the type $\int_{-1}^{1} 1\left(x-x_{1}\right)^{3} d x$. Hence, if one of the expressions $M_{i}, i=1,2,3$ vanish in the interval $[-1,1]$ then the integrand will be not integrable. Thus, to guarantee the convergence of the integrals, we are looking for such conditions on variables $\chi_{1}, \chi_{2}, \chi_{3}$ which give nonzero values for $M_{i}$ within of the corresponding interval. The necessary and sufficient conditions of the convergence of the integrals (4.2) are as follows

$$
\chi_{1}>-1, \quad \chi_{2}>-1, \quad \chi_{3}>-\left(\sqrt{1+\chi_{1}}+\sqrt{1+\chi_{2}}\right)^{2} .
$$

The detailed proof of these conditions can be found in Appendix II. They are also important in the numerical solution of our system of differential equations and these conditions must be tested on each step of the Runge-Kutta method.

An important question is related to the choice of a numerical method for calculation of the integrals. It can be shown that the most appropriate method is the using of the Chebyshev quadrature formula. The question of the number of divisions of the integration interval is another important one. In our calculations, we used the division to 1024 subintervals, which was found as the best choice from the point of view of the accuracy and needed time of the calculation. On the other hand, in [7], the division to the 128 subintervals was used. We suppose that this fact could lead to the difference between our and their results because the division to 128 subintervals may not be sufficient in some critical situations.

To find the borderline dimension it is enough to use the bisection method. Our results were calculated with the accuracy of 0.005 . The same accuracy was supposed in [7] but, as can be seen from their results, this accuracy was not achieved by them even in the isotropic limit where the exact result exists.

From the numeric calculations point of view, the problem is rather timeconsuming, i.e., the calculations take relatively long time. Therefore, the question of using modern computational methods arises. In what follows, we shall analyze a possible speed up of calculations based on the utilization of the parallel programming methods using the Message Passing Interface (MPI) (see, e.g., $[10,20])$. Let us discuss this problem in more detail. First of all, we have to solve our system of differential equations at large number of points. For example, Fig. 1 was obtained by using results on the lattice of the size $16 \times 16$ in the plane of anisotropy parameters $\alpha_{1}$ and $\alpha_{2}$, i.e., we had to repeat the numerical procedure 256 times. 
Suppose first that we have to calculate the borderline dimension $d_{c}$ for one concrete value of the parameters $\alpha_{1}$ and $\alpha_{2}$. How can the parallel algorithms help in this situation? To find the borderline dimension with a precision $\Delta d$ when the starting interval for $d$ has the length $l$ one has to carry out a prescribed number of calculations $n$. Using one-processor computer the best way how to find the $d_{c}$ is to use the bisection method. In this case, the result is obtained after $n=\left\lceil\log _{2}(l / \Delta d)\right\rceil$ calculations $(\lceil x\rceil$ means the smallest integer greater or equal to $x$ ). On the other hand, in the case of the multi-processor computer with $m$ processors, one can divide the interval into $m+1$ subintervals and carry out the calculations in $m$ points of the division at the same time. Thus, the result is obtained after $n=\left\lceil\log _{m+1}(l / \Delta d)\right\rceil$ serial calculations (of course, in this case, the total number of calculations is larger but the total time of the calculations is shorter).

Table 1. The number of needed serial calculations $n$ as a function of the number of processors $m$ for the special case: $l=1$ and $\Delta d=0.005$.

\begin{tabular}{ccccccc}
\hline$m$ & 1 & 2 & 3,4 & $5-13$ & $14-198$ & $\geq 199$ \\
\hline$n$ & 8 & 5 & 4 & 3 & 2 & 1 \\
\hline
\end{tabular}

Let us demonstrate this speed-up by an example. Suppose that $l=1$, and $\Delta d=0.005$. The results are shown in Tab. 1 . The table shows the effective numbers of processors which are 1,2,3,5,14, and 199. If we suppose that the calculations take the same time for all values of dimension $d$ then $n$ is directly related to the time of calculation. For example, the calculation with three processors (the same holds also for four processors) is two times shorter then calculation with one processor, see Tab. 1 . On the other hand, the calculation takes the same time for the computations with 3 and 4 processors. The same is held for the computers with number of processors from the intervals $m=[5,13], m=[14,198]$, and $m=[199, \infty)$. Therefore, our conclusion is the following: if one needs to do only one computational process (in our case, it means to find one borderline dimension $d_{c}$ for concrete value of the parameters of the model) then it is appropriate to use the advantage of the parallel computing.

Now let us analyze the situation when one needs to calculate the borderline dimension $d_{c}$ as a function of the anisotropy parameters (as it is in our case). Thus, it is necessary to carry out two or more independent calculations for different values of the parameters of the model. The simplest situation occurs when the number of independent calculations are much larger than the number of processors. Because this is our case, we shall analyze it in detail. The situation is shown in Fig. 3, where the total number of computational processes $N$ is shown as a function of the number of processors $m$ and of the desired precision $\Delta d$. It is seen immediately that the most effective utilization of the processors is to give to each processor to calculate independent borderline dimension $d_{c}$ alone. 


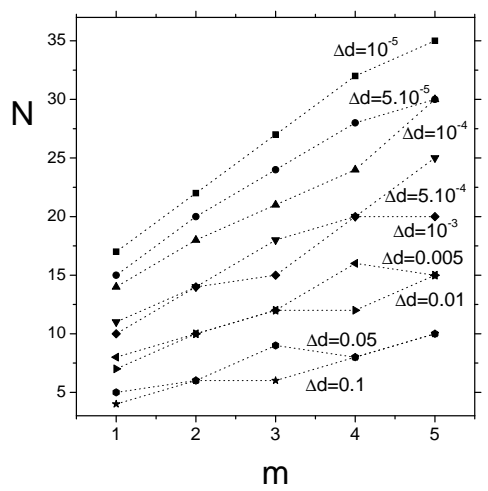

Figure 3. The dependence of the total number of calculational processes as a function of the number of processors $m$ and the precision $\Delta d$. The length of the initial interval is $l=1$.

We have analyzed two special cases, namely, the case with one independent calculation and the case with the large number of independent calculations. The situation becomes more complicated when one needs to carry out the number of calculations which is comparable to the number of processors. But each such situation needs special analysis and we shall not analyze it here.

In concrete calculations we used advantages of the parallel programming. Our situation is the case with a large number of independent calculations, therefore, each processor has calculated borderline dimension for defined values of the parameters of the model.

\section{Conclusion}

By using the field-theoretic RG method the influence of the uniaxial anisotropy on the stability of the Kolmogorov scaling regime in fully developed turbulence was investigated. The stability of the regime is defined by the very existence of the IR stable fixed point. The fixed point was found numerically by solving the corresponding differential RG equations. It was shown that the earlier results obtained in [7] as well as their conclusions about the dependence of the borderline dimension $d_{c}$ as a function of the anisotropy parameters $\alpha_{1,2}$ are not precise enough. We have found that the stability of the three-dimensional scaling regime is destroyed only in the case of rather large (in the sense of the absolute value) and special values of the anisotropy parameters. We have also 
analyzed the optimal way how to calculate the numerical problem by using the parallel programming methods.

\section{Acknowledgments}

E.J. is thankful to J. Buša for discussion. M.S. gratefully acknowledges the hospitality of the Laboratory of Theoretical Physics at JINR, Dubna. The work was supported in part by VEGA grant 6193 of Slovak Academy of Sciences, by Science and Technology Assistance Agency under contract No. APVT51027904, and by grants RFFI-RFBR 05-01-00645, 04-01-00490, 04-01-81011, and $05-02-17603$.

\section{Appendix I}

The explicit form of the functions $a_{i}, i=1, \ldots, 4$ is as follows

$$
\begin{aligned}
& a_{i}=\frac{1}{4} \int_{-1}^{1} \frac{\left(1-x^{2}\right)^{(d-3) / 2}}{\left(M_{1} M_{2} M_{3}\right)^{3}} b_{i} d x, \quad i=1, \ldots, 4, \\
& b_{1}=c_{1,1}+4 M_{2} M_{3} x^{2} x_{1}^{2}\left(c_{1,2}+c_{1,3}\right) \\
& +M_{1} x^{2} x_{1}\left(c_{1,4}+M_{2} M_{3}^{2} r_{4} x_{1} c_{1,5}+M_{2}^{3} c_{1,6}-2 M_{2}^{2} M_{3}^{2} c_{1,7}\right), \\
& b_{2}=c_{2,1}-M_{1}^{2} M_{2}^{3} r_{1}\left(c_{2,2}+c_{2,3}\right)-4 M_{2} M_{3} x_{1}\left(f_{3}+x_{1}\right) c_{2,4} \\
& -M_{1}\left(-2 M_{3}^{3} r_{4}^{2} w_{1} x_{1}^{2}\left(f_{3}+x_{1}\right) y_{4}+M_{2}^{3}\left(2 x_{1}^{2} c_{2,5}-2 f_{3} c_{2,6}+d_{1} M_{3} r_{1} r_{4}^{2} x_{1} y_{7}\right)\right. \\
& \left.+2 M_{2}^{2} M_{3}^{2}\left(2 \alpha_{2} M_{3} c_{2,7}+c_{2,8}\right)+M_{2} M_{3}^{2} r_{4}\left(c_{2,9}+c_{2,10}\right)\right), \\
& b_{3}=c_{3,1}+M_{1}^{2} M_{2}^{3} r_{1} c_{3,2}+4 M_{2} M_{3}\left(c_{3,3}+M_{2} M_{3} r_{4}\left(c_{3,4}+x_{1} c_{3,5}\right)\right. \\
& \left.+M_{2}^{2}\left(c_{3,6}+x_{1} c_{3,7}\right)\right)+M_{1}\left(c_{3,8}-2 M_{2}^{2} M_{3}^{2}\left(c_{3,9}+2 f_{3} c_{3,10}+c_{3,11}\right)\right. \\
& \left.+M_{2} M_{3}^{2} r_{4}\left(-x_{1} c_{3,12}-4 f_{3} c_{3,13}\right)+M_{2}^{3}\left(-d_{1} c_{3,14}+2 f_{3} c_{3,15}-2 x_{1}^{2} c_{3,16}\right)\right) \text {, } \\
& \frac{b_{4}}{2}=c_{4,1}-2 c_{4,2} M_{1}^{2} M_{2}^{3} r_{1}+2 M_{2} M_{3}\left(-c_{4,7}+\left(c_{4,5}-c_{4,6} d_{1}\right) M_{2}^{2}\right. \\
& \left.+\left(c_{4,3}-c_{4,4} d_{1}\right) M_{2} M_{3} r_{4}\right)+M_{1}\left(\left(d_{1}\left(c_{4,10}+c_{4,11} f_{1}\right)+c_{4,9} f_{2}+3 c_{4,8} f_{3}\right) M_{2}^{3}\right. \\
& +2\left(d_{1}\left(c_{4,20}+2 c_{4,19} f_{1}\right)+c_{4,18} f_{2}+3 c_{4,17} f_{3}\right) M_{2}^{2} M_{3}^{2} \\
& \left.+\left(d_{1}\left(c_{4,15}+2 c_{4,16} f_{1}\right)+c_{4,14} f_{2}+3 c_{4,13} f_{3}\right) M_{2} M_{3}^{2} r_{4}-c_{4,12} M_{3}^{3} r_{4}^{2} x_{1} y_{4}\right), \\
& c_{1,1}=4 M_{1}^{2} M_{2}^{3} r_{1} x^{2} x_{1}\left(-d_{1} M_{3} r_{4}+\left(-\chi_{2} M_{3}+r_{4}\left(1+3 M_{3}\right)\right) x_{1}\right) \\
& +M_{1}^{3} M_{2}^{3} r_{1}\left(d_{1} M_{3}\left(d_{-1}-3 x_{1}\right)+2\left(1+2 M_{3}\right) x_{1}^{2}\right), \\
& c_{1,2}=-M_{3}^{2} r_{4}^{2} x_{1}\left(r_{1}+\alpha_{2} x_{1}\right)+M_{2}^{2}\left(-4 \alpha_{2} M_{3}^{2}+r_{1} r_{4}\left(2 M_{3}-r_{4}\left(1+w_{1}\right) x_{1}\right)\right) \text {, } \\
& c_{1,3}=M_{2} M_{3} r_{4}\left(4 \alpha_{2} M_{3} x_{1}+r_{1}\left(2 M_{3}-r_{4}\left(2+w_{1}\right) x_{1}\right)\right), \\
& c_{1,4}=-2 M_{3}^{3} r_{4}^{2} w_{1} x_{1}^{2}\left(r_{1}+\alpha_{2} x_{1}\right) \text {, }
\end{aligned}
$$




$$
\begin{aligned}
& c_{1,5}=\alpha_{2} M_{3} x_{1}\left(4\left(w_{1}+h_{2} x_{1}\right)+r_{4} y_{1}\right)+r_{1}\left(4 \chi_{2} M_{3} x_{1}+r_{4}\left(-4 w_{1} x_{1}+M_{3} y_{1}\right)\right) \text {, } \\
& c_{1,6}=d_{1} M_{3}\left(4 \alpha_{2} M_{3}^{2}+r_{1} r_{4}\left(-2 M_{3}+r_{4} x_{1}\right)\right) \\
& -2 x_{1}\left(8 \alpha_{2} M_{3}^{3}+r_{1} r_{4}\left(-4 M_{3}^{2}+r_{4} x_{1}-2 M_{3}\left(1+w_{1}-y_{2}\right)\right)\right), \\
& c_{1,7}=d_{1} r_{4}\left(2 \alpha_{2} M_{3} x_{1}+r_{1}\left(M_{3}-r_{4} x_{1}\right)\right) \\
& -4 x_{1}\left(r_{1} r_{4}\left(M_{3}-y_{2}\right)+\alpha_{2} M_{3}\left(r_{4} x_{1}+y_{2}\right)\right) \text {, } \\
& c_{2,1}=2 M_{1}^{3} M_{2}^{3} r_{1}\left(f_{3}-d_{1} f_{1} M_{3}+2 f_{3} M_{3}\right) \text {, } \\
& c_{2,2}=-4 f_{3} M_{3} r_{4}\left(-2+3 x^{2}\right)+d_{1} M_{3} r_{4}\left(d_{-1}-4 f_{1}+3 f_{1} x^{2}-5 x_{1}\right), \\
& c_{2,3}=4 f_{3}\left(-\chi_{2} M_{3}+r_{4}\right) x_{1}+4\left(-\chi_{2} M_{3}+r_{4}+2 M_{3} r_{4}\right) x_{1}^{2} \text {, } \\
& c_{2,4}=-4 \alpha_{2} M_{2} M_{3}^{2}\left(M_{2}-r_{4} x_{1}\right) \\
& -r_{4}\left(M_{2} r_{1}\left(-2 M_{1} M_{3}+r_{4}\left(M_{1}+M_{3}+M_{1} w_{1}\right) x_{1}\right)+M_{3}^{2} r_{4} x_{1} y_{4}\right), \\
& c_{2,5}=-4 \alpha_{2} M_{3}^{3}+r_{1} r_{4}\left(-r_{4}\left(M_{3} x_{4}+x_{1}\right)+2 M_{3}\left(1+M_{3}+w_{1}+\chi_{2} x_{1}\right)\right) \text {, } \\
& c_{2,6}=4 \alpha_{2} M_{3}^{3} x_{2}+r_{1} r_{4}\left(r_{4}\left(x_{1}^{2}+M_{3} y_{3}\right)-2 M_{3}\left(M_{3} x_{2}+x_{1}\left(1+y_{5}\right)\right)\right) \text {, } \\
& c_{2,7}=x_{1}^{2}\left(-2 \chi_{2} x_{1}+r_{4}\left(x_{4}+x_{1}\right)\right) \\
& +f_{3}\left(-2 \chi_{2} x_{1}^{2}+r_{4}\left(1+4 x^{4}+x_{1}-2 x^{2}\left(3+x_{1}\right)\right)\right) \text {, } \\
& c_{2,8}=2 f_{3} r_{1} r_{4}\left(M_{3} x_{2}+2 \chi_{2} x_{1}^{2}-r_{4} y_{3}\right) \\
& +r_{4} x_{1}\left(2 r_{1} x_{1}\left(M_{3}-r_{4} x_{4}+2 \chi_{2} x_{1}\right)+d_{1} y_{6} y_{7}\right) \text {, } \\
& c_{2,9}=-2 r_{1}\left(-2 \chi_{2} M_{3} x_{1}^{3}+r_{4}\left(x_{1}^{2}\left(M_{3} x_{4}+2 w_{1}\left(f_{3}+x_{1}\right)\right)+f_{3} M_{3} y_{3}\right)\right) \text {, } \\
& c_{2,10}=M_{3} x_{1}\left(\alpha_{2}\left(4 f_{3} w_{1} x_{1}-2 r_{4} x_{4} x_{1}^{2}-2 f_{3} r_{4} y_{3}+4 x_{1}^{2} y_{5}\right)\right. \\
& \left.+y_{4}\left(4 f_{3} \chi_{2} x_{1}+d_{1} r_{4} y_{7}\right)\right) \text {, } \\
& c_{3,1}=M_{1}^{3} M_{2}^{3} r_{1}\left(-d_{1} f_{1} M_{3}+2 f_{3}\left(1+\chi_{1}+2 M_{3}\right)+2 \chi_{1} x_{1}^{2}\right), \\
& c_{3,2}=-4\left(-\chi_{2} M_{3}+r_{4}+3 M_{3} r_{4}+\chi_{3} M_{3} x^{2}-\chi_{1} r_{4} x^{2}\right) x_{1}^{2} \\
& -d_{1}\left(d_{-1} M_{3} r_{4}+f_{1} M_{3} r_{4}\left(-2+x^{2}\right)+\left(-5 M_{3} r_{4}-4 \chi_{3} M_{3} x^{2}\right.\right. \\
& \left.\left.+4 \chi_{1} r_{4} x^{2}\right) x_{1}\right)+4 f_{3}\left(M_{3}\left(-\chi_{3} x^{2}+\chi_{2} x_{1}\right)+r_{4}\left(-3 M_{3} x_{1}+y_{8}\right)\right) \text {, } \\
& c_{3,3}=-M_{3}^{2} r_{4}^{2} x_{1} y_{4}\left(-d_{1} \chi_{1} x^{2} x_{1}+f_{3} y_{8}+x_{1}^{2} y_{9}\right) \text {, } \\
& c_{3,4}=f_{3}\left(4 \alpha_{2} M_{3} x_{1} y_{8}+r_{1}\left(-r_{4} x_{1}\left(-2-w_{1}+x^{2}\left(2+2 \chi_{1}+w_{1}+w_{2}\right)\right)+2 M_{3} y_{8}\right)\right) \text {, } \\
& c_{3,5}=-d_{1} x^{2}\left(2 \chi_{1}\left(2 \alpha_{2} M_{3} x_{1}+r_{1}\left(M_{3}-r_{4} x_{1}\right)\right)-r_{1} r_{4} x_{1} w_{2}\right) \\
& +x_{1}\left(4 \alpha_{2} M_{3} x_{1} y_{9}+r_{1}\left(r_{4} x_{1}\left(w_{1}-x^{2} w_{2}-2 y_{9}\right)+2 M_{3} y_{9}\right)\right) \text {, } \\
& c_{3,6}=-f_{3}\left(4 \alpha_{2} M_{3}^{2} y_{8}+r_{1} r_{4}\left(r_{4} x_{1}\left(-1-w_{1}+x^{2}\left(1+\chi_{1}+w_{1}+w_{2}\right)\right)-2 M_{3} y_{8}\right)\right) \text {, } \\
& c_{3,7}=d_{1} x^{2}\left(\chi_{1}\left(4 \alpha_{2} M_{3}^{2}+r_{1} r_{4}\left(-2 M_{3}+r_{4} x_{1}\right)\right)+r_{1} r_{4}^{2} x_{1} w_{2}\right) \\
& +x_{1}\left(-4 \alpha_{2} M_{3}^{2} y_{9}+r_{1} r_{4}\left(r_{4} x_{1}\left(w_{1}-x^{2} w_{2}-y_{9}\right)+2 M_{3} y_{9}\right)\right), \\
& c_{3,8}=-2 M_{3}^{3} r_{4}^{2} x_{1}\left(f_{3}\left(-w_{1} x_{1}+x^{2} w_{2}\right)-x_{1}\left(w_{1} x_{1}+x^{2}\left(d+x^{2}\right) w_{2}\right)\right) y_{4} \text {, }
\end{aligned}
$$




$$
\begin{aligned}
& c_{3,9}=2 x_{1}^{2}\left(r_{1} r_{4}\left(3 M_{3}-2 r_{4} x_{3}+2 \chi_{2} x_{1}-2 \chi_{3} x^{2} x_{1}\right)\right. \\
& \left.+\alpha_{2} M_{3}\left(-2\left(\chi_{2}-\chi_{3} x^{2}\right) x_{1}+r_{4}\left(2 x_{3}+3 x_{1}\right)\right)\right), \\
& c_{3,10}=\alpha_{2} M_{3}\left(2 x_{1}\left(\chi_{3} x^{2}-\chi_{2} x_{1}\right)+r_{4}\left(2+4 x^{4}+3 x_{1}-2 x^{2}\left(4+x_{1}\right)\right)\right) \\
& +r_{1} r_{4}\left(3 M_{3}-2 M_{3} x^{2}-2 \chi_{3} x^{2} x_{1}+2 \chi_{2} x_{1}^{2}-2 r_{4} y_{10}\right), \\
& c_{3,11}=d_{1}\left(x _ { 1 } \left(\alpha_{2} M_{3}\left(r_{4}\left(-x_{5}-4 x_{1}\right)-4 \chi_{3} x^{2} x_{1}\right)\right.\right. \\
& \left.\left.+r_{1} r_{4}\left(-4 M_{3}+r_{4} x_{5}+4 \chi_{3} x^{2} x_{1}\right)\right)+d_{-1} M_{3} r_{4} y_{4}+f_{1} M_{3} r_{4}\left(-2+x^{2}\right) y_{4}\right) \text {, } \\
& c_{3,12}=d_{1}\left(\alpha_{2} M_{3} x_{1}\left(r_{4} x_{5}+4 x^{2}\left(\chi_{3} x_{1}+w_{2}\right)\right)+r_{1}\left(4 \chi_{3} M_{3} x^{2} x_{1}\right.\right. \\
& \left.\left.+r_{4}\left(M_{3} x_{5}-4 x^{2} x_{1} w_{2}\right)\right)\right)-4 x_{1}\left(\alpha _ { 2 } M _ { 3 } x _ { 1 } \left(-w_{1}+r_{4} x_{3}-\chi_{2} x_{1}+\chi_{3} x^{2} x_{1}\right.\right. \\
& \left.\left.+x^{2} w_{2}\right)+r_{1}\left(M_{3}\left(-\chi_{2}+\chi_{3} x^{2}\right) x_{1}+r_{4}\left(M_{3} x_{3}+w_{1} x_{1}-x^{2} x_{1} w_{2}\right)\right)\right), \\
& c_{3,13}=\alpha_{2} M_{3} x_{1}\left(w_{1} x_{1}-r_{4} y_{10}-x^{2}\left(\chi_{3} x_{1}+w_{2}\right)\right) \\
& -r_{1}\left(\chi_{3} M_{3} x^{2} x_{1}+r_{4}\left(w_{1} x_{1}^{2}+M_{3} y_{10}-x^{2} x_{1} w_{2}\right)\right)+\chi_{2} M_{3} x_{1}^{2} y_{4}, \\
& c_{3,14}=2 d_{-1} M_{3}^{2}\left(-2 \alpha_{2} M_{3}+r_{1} r_{4}\right)+2 f_{1} M_{3}^{2}\left(-2 \alpha_{2} M_{3}+r_{1} r_{4}\right)\left(-2+x^{2}\right) \\
& +x_{1}\left(16 \alpha_{2} M_{3}^{3}+r_{1} r_{4}\left(r_{4}\left(M_{3} x_{5}-2 \chi_{1} x^{2} x_{1}\right)\right.\right. \\
& \left.\left.+4 M_{3}\left(-2 M_{3}+x^{2}\left(\chi_{1}+\chi_{3} x_{1}+w_{2}\right)\right)\right)\right) \text {, } \\
& c_{3,15}=4 \alpha_{2} M_{3}^{3}\left(3-2 x^{2}\right)+r_{1} r_{4}\left(M_{3}^{2}\left(-6+4 x^{2}\right)\right. \\
& \left.+2 M_{3}\left(\chi_{1} x^{2}-x_{1}+\chi_{3} x^{2} x_{1}-w_{1} x_{1}-\chi_{2} x_{1}^{2}+r_{4} y_{10}+x^{2} w_{2}\right)-r_{4} x_{1} y_{8}\right) \text {, } \\
& c_{3,16}=-12 \alpha_{2} M_{3}^{3}+r_{1} r_{4}\left(2 M_{3}\left(3 M_{3}+w_{1}+\chi_{2} x_{1}-\chi_{3} x^{2} x_{1}-x^{2} w_{2}-y_{9}\right)\right. \\
& \left.-r_{4}\left(2 M_{3} x_{3}-x_{1} y_{9}\right)\right) \text {, } \\
& c_{4,1}=M_{1}^{3} M_{2}^{3}\left(3 f_{3} \chi_{1}+f_{2}\left(1+\chi_{1}+2 M_{3}\right)\right) r_{1}, \\
& c_{4,2}=-3 f_{3}\left(2 \chi_{2} M_{3}-\chi_{3} M_{3} x^{2}+r_{4}\left(-5 M_{3}+x_{8}\right)\right) \\
& +f_{2}\left(M_{3}\left(\chi_{3} x^{2}+\chi_{2} x_{7}\right)-r_{4}\left(M_{3}\left(-5+3 x^{2}\right)+x_{9}\right)\right) \\
& +d_{1}\left(f_{1}\left(M_{3}\left(\chi_{2}+\chi_{3}-2 \chi_{3} x^{2}\right)+r_{4}\left(-2 M_{3}+x_{10}\right)\right)+\chi_{3} M_{3} x_{1}-\chi_{1} r_{4} x_{1}\right), \\
& c_{4,3}=3 f_{3}\left(4 \alpha_{2} M_{3} x_{8} x_{1}+r_{1}\left(2 M_{3} x_{8}+r_{4} x_{1}\left(2 w_{1}-2 x_{8}-x^{2} w_{2}\right)\right)\right) \\
& +f_{2}\left(4 \alpha_{2} M_{3} x_{9} x_{1}+r_{1}\left(2 M_{3} x_{9}-r_{4} x_{1}\left(-2\left(2+w_{1}\right)\right.\right.\right. \\
& \left.\left.\left.+x^{2}\left(2+2 \chi_{1}+w_{1}+w_{2}\right)\right)\right)\right) \text {, } \\
& c_{4,4}=x_{1}\left(-2 \chi_{1}\left(M_{3} r_{1}+2 \alpha_{2} M_{3} x_{1}-r_{1} r_{4} x_{1}\right)+r_{1} r_{4} x_{1} w_{2}\right)+f_{1}\left(4 \alpha_{2} M_{3} x_{10} x_{1}\right. \\
& \left.+r_{1}\left(2 M_{3} x_{10}+r_{4} x_{1}\left(2+2 \chi_{1}-4 \chi_{1} x^{2}+w_{1}+x_{2} w_{2}\right)\right)\right), \\
& c_{4,5}=3 f_{3}\left(-4 \alpha_{2} M_{3}^{2} x_{8}+r_{1} r_{4}\left(2 M_{3} x_{8}-r_{4} x_{1}\left(-2 w_{1}+x_{8}+x^{2} w_{2}\right)\right)\right) \\
& +f_{2}\left(-4 \alpha_{2} M_{3}^{2} x_{9}+r_{1} r_{4}\left(2 M_{3} x_{9}-r_{4} x_{1}\left(-2\left(1+w_{1}\right)\right.\right.\right. \\
& \left.\left.\left.+x^{2}\left(1+\chi_{1}+w_{1}+w_{2}\right)\right)\right)\right) \text {, } \\
& c_{4,6}=x_{1}\left(\chi_{1}\left(4 \alpha_{2} M_{3}^{2}+r_{1} r_{4}\left(-2 M_{3}+r_{4} x_{1}\right)\right)+r_{1} r_{4}^{2} x_{1} w_{2}\right) \\
& +f_{1}\left(-4 \alpha_{2} M_{3}^{2} x_{10}+r_{1} r_{4}\left(2 M_{3} x_{10}+r_{4} x_{1}\left(-x_{10}+w_{1}+x_{2} w_{2}\right)\right)\right) \text {, }
\end{aligned}
$$




$$
\begin{aligned}
& c_{4,7}=M_{3}^{2} r_{4}^{2} x_{1}\left(-d 1 f_{1} x_{10}+3 f_{3} x_{8}+f_{2} x_{9}+d 1 \chi_{1} x_{1}\right) y_{4}, \\
& c_{4,8}=16 \alpha_{2} M_{3}^{3}+r_{1} r_{4}\left(r_{4}\left(M_{3} x_{6}-x_{8} x_{1}\right)\right. \\
& \left.+2 M_{3}\left(-4 M_{3}-2 w_{1}+x_{8}-2 \chi_{2} x_{1}+\chi_{3} x^{2} x_{1}+x^{2} w_{2}\right)\right), \\
& c_{4,9}=-8 \alpha_{2} M_{3}^{3} x_{7}+r_{1} r_{4}\left(r_{4}\left(-x_{9} x_{1}+M_{3} y_{13}\right)\right. \\
& \left.+2 M_{3}\left(\chi_{1} x^{2}+\left(1+2 M_{3}+w_{1}\right) x_{7}+\chi_{3} x^{2} x_{1}-\chi_{2} y_{11}+x^{2} w_{2}\right)\right), \\
& c_{4,10}=r_{1} r_{4}\left(d_{-1} M_{3} r_{4}+x_{1}\left(2 \chi_{1} M_{3}-5 M_{3} r_{4}+2 \chi_{3} M_{3} x_{1}-\chi_{1} r_{4} x_{1}+2 M_{3} w_{2}\right)\right) \text {, } \\
& c_{4,11}=-8 \alpha_{2} M_{3}^{3}+r_{1} r_{4}\left(4 M_{3}^{2}-r_{4}\left(x_{1}+\chi_{1} y_{12}\right)\right. \\
& \left.+2 M_{3}\left(2 \chi_{3} x^{4}-x_{10}+r_{4} x_{11}+w_{1}+\chi_{3} x_{3}+\chi_{2} x_{1}+x_{2} w_{2}\right)\right), \\
& c_{4,12}=-6 f_{3} w_{1}+f_{2} w_{1} x_{7}+f_{2} x^{2} w_{2}+3 f_{3} x^{2} w_{2}+d 1\left(x_{1} w_{2}+f_{1}\left(w_{1}+x_{2} w_{2}\right)\right), \\
& c_{4,13}=\alpha_{2} M_{3} x_{1}\left(-4 w_{1}+r_{4} x_{6}+2 x^{2}\left(\chi_{3} x_{1}+w_{2}\right)\right) \\
& +r_{1}\left(2 \chi_{3} M_{3} x^{2} x_{1}+r_{4}\left(M_{3} x_{6}+4 w_{1} x_{1}-2 x^{2} x_{1} w_{2}\right)\right)-4 \chi_{2} M_{3} x_{1} y_{4}, \\
& c_{4,14}=\alpha_{2} M_{3}\left(-2 w_{1} y_{11}+r_{4} x_{1} y_{13}+2 x^{2} x_{1}\left(\chi_{3} x_{1}+w_{2}\right)\right) \\
& +r_{1}\left(2 \chi_{3} M_{3} x^{2} x_{1}+r_{4}\left(M_{3} y_{13}-2 x_{1}\left(w_{1} x_{7}+x^{2} w_{2}\right)\right)\right)-2 \chi_{2} M_{3} y_{11} y_{4}, \\
& c_{4,15}=d_{-1} M_{3} r_{4} y_{4}-x_{1}\left(r_{1} r_{4}\left(5 M_{3}+2 x_{1} w_{2}\right)+M_{3} x_{1}\left(5 \alpha_{2} r_{4}-2 \alpha_{2} w_{2}-2 \chi_{3} y_{4}\right)\right) \text {, } \\
& c_{4,16}=r_{1} r_{4}\left(M_{3} x_{11}-x_{1}\left(w_{1}+x_{2} w_{2}\right)\right) \\
& +M_{3}\left(\alpha_{2} r_{4} x_{11} x_{1}+\alpha_{2} w_{1} x_{1}+\alpha_{2} y_{12} w_{2}+\chi_{2} x_{1} y_{4}+\chi_{3} y_{12} y_{4}\right) \text {, } \\
& c_{4,17}=r_{1} r_{4}\left(-4 M_{3}+r_{4} x_{6}-4 \chi_{2} x_{1}+2 \chi_{3} x^{2} x_{1}\right) \\
& -\alpha_{2} M_{3}\left(r_{4} x_{6}-4 \chi_{2} x_{1}+4 r_{4} x_{1}+2 \chi_{3} x^{2} x_{1}\right), \\
& c_{4,18}=r_{1} r_{4}\left(2 M_{3} x_{7}+2 \chi_{3} x^{2} x_{1}-2 \chi_{2} y_{11}+r_{4} y_{13}\right) \\
& -\alpha_{2} M_{3}\left(2 \chi_{3} x^{2} x_{1}+2 r_{4} x_{2} x_{1}-2 \chi_{2} y_{11}+r_{4} y_{13}\right) \text {, } \\
& c_{4,19}=r_{1} r_{4}\left(M_{3}+r_{4} x_{11}+\chi_{2} x_{1}+\chi_{3} y_{12}\right) \\
& -\alpha_{2} M_{3}\left(r_{4} x_{11}+\chi_{2} x_{1}-r_{4} x_{1}+\chi_{3} y_{12}\right) \\
& c_{4,20}=\left(d_{-1} r_{4}+x_{1}\left(-5 r_{4}+2 \chi_{3} x_{1}\right)\right) y_{6}, \\
& M_{1}=2\left(1+\chi_{1} x^{2}\right)+\left(\chi_{2}+x^{2} \chi_{3}\right)\left(1-x^{2}\right), \\
& M_{2}=1+\chi_{1} x^{2}+\left(\chi_{2}+x^{2} \chi_{3}\right)\left(1-x^{2}\right), \quad M_{3}=1+\chi_{1} x^{2}, \\
& r_{1}=1+\alpha_{1} x^{2}, \quad r_{4}=\chi_{2}+\chi_{3} x^{2}, \quad f_{1}=x^{2} d-1, \\
& f_{2}=-(d+2) x^{4}+(d+3) x^{2}-1, \quad f_{3}=(d+4)(d+2) x^{4}-6(d+2) x^{2}+3, \\
& w_{1}=1+\chi_{2}+\chi_{3} x^{4}, \quad w_{2}=\chi_{1}-\chi_{2}+\chi_{3}\left(1-2 x^{2}\right), \\
& y_{1}=-3+d+8 x^{2}, \quad y_{2}=r_{4}\left(1-2 x^{2}\right)-\chi_{2}\left(1-x^{2}\right), \quad y_{3}=1-6 x^{2}+4 x^{4}, \\
& y_{4}=r_{1}+\alpha_{2} x_{1}, \quad y_{5}=w_{1}+\chi_{2} x_{1}, \quad y_{6}=r_{1} r_{4}-\alpha_{2} M_{3}, \quad y_{7}=1-3 x^{2}+f_{1} x_{1} \text {, } \\
& y_{8}=-1+\left(1+\chi_{1}\right) x^{2}, \quad y_{9}=-1+\chi_{1} x^{2}, \quad y_{10}=1-4 x^{2}+2 x^{4} \text {, }
\end{aligned}
$$




$$
\begin{aligned}
& y_{11}=2-3 x^{2}+x^{4}, \quad y_{12}=1-3 x^{2}+2 x^{4}, \quad y_{13}=3-12 x^{2}+4 x^{4}, \\
& d_{1}=d+1, \quad d_{-1}=d-1, \\
& x_{1}=1-x^{2}, \quad x_{2}=1-2 x^{2}, \quad x_{3}=1-3 x^{2}, \quad x_{4}=1-4 x^{2}, \\
& x_{5}=1-5 x^{2}, \quad x_{6}=3-10 x^{2}, \quad x_{7}=-2+x^{2}, \quad x_{8}=\chi_{1} x^{2}-2, \\
& x_{9}=\left(1+\chi_{1}\right) x^{2}-2, \quad x_{10}=\chi_{1}\left(2 x^{2}-1\right)-1, \quad x_{11}=-3+5 x^{2} .
\end{aligned}
$$

\section{Appendix II}

In this Appendix we shall prove the necessary and sufficient conditions needed for the convergence of integrals (4.2).

Theorem 1. If the expressions $M_{i}, i=1,2,3$ (see Appendix I) are nonzero at each point $x \in[-1,1]$ then they are positive on the whole interval.

Proof. Expressions $M_{i}, i=1,2,3$ are continuous functions with respect to $x$ within the interval $x \in[-1,1]$. On the other hand, $M_{3}(0)=1>0$. If one supposes that there exists $y \in[-1,1]$ such that $M_{3}(y)<0$ then according to the property of continuity there must exist a point at which the function is equal to zero. But it is in a contradiction with the assumption of the theorem. Thus, $M_{3}(x)>0$ for all $x \in[-1,1]$. Because $M_{2}(1)=1+\chi_{1}=M_{3}(1)>0$, then using the same arguments we come to the same conclusion, namely, $M_{2}(x)>0$ for all $x \in[-1,1]$. Finally, because $M_{1}=M_{2}+M_{3}$, then $M_{1}(x)$ is also positive for all $x \in[-1,1]$. Thus, the theorem is proved.

Now we are able to prove the necessary and sufficient conditions of the convergence which are the contents of the following theorem.

Theorem 2 [Necessary and sufficient conditions of the convergence of integrals (4.2)]. Expressions $M_{i},(i=1,2,3)$ are nonzero for each $x \in$ $[-1,1]$ if and only if the following conditions are satisfied:

i) $\chi_{1} \in(-1, \infty)$;

ii) $\chi_{2} \in(-1, \infty)$;

iii) $\chi_{3} \in\left(-\left(\sqrt{1+\chi_{1}}+\sqrt{1+\chi_{2}}\right)^{2}, \infty\right)$.

Proof. First we prove that if expressions $M_{i}$ are nonzero for each $x \in[-1,1]$ then the conditions $i$ ), ii), and iii) are satisfied. Suppose that the implication is not true, i.e., expressions $M_{i}$ are nonzero and at the same time some of the conditions $i$ ), ii) and iii) are not satisfied. We shall show in items $a$ ), b), and $c$ ) that when the conditions for parameters $\chi_{1}, \chi_{2}, \chi_{3}$ are not satisfied then one comes to a contradiction.

a) Let us suppose that $\chi_{1} \leq-1$. It is enough to take $\hat{x}=\sqrt{-1 / \chi_{1}}$, and it is evident that $0<\sqrt{-1 / \chi_{1}} \leq 1$, therefore $\hat{x} \in(0,1] \subseteq[-1,1]$. But 


$$
M_{3}(\hat{x})=M_{3}\left(\sqrt{-\frac{1}{\chi_{1}}}\right)=1+\chi_{1}\left(\sqrt{-\frac{1}{\chi_{1}}}\right)^{2}=0,
$$

which is in contradiction with the assumption of the theorem.

b) Suppose that $\chi_{2} \leq-1$, then $M_{2}(0)=1+\chi_{2} \leq 0$, and according to the

Theorem 1 it is in contradiction with the assumption of the theorem.

c) In the end, suppose that $\chi_{3} \leq-\left(\sqrt{1+\chi_{1}}+\sqrt{1+\chi_{2}}\right)^{2}$. Therefore,

$$
\chi_{3}+\left(\sqrt{1+\chi_{1}}+\sqrt{1+\chi_{2}}\right)^{2} \leq 0 .
$$

At the same time, as was already proved in items $a$ ) and $b), \chi_{1}>-1$ and $\chi_{2}>-1$. Take

$$
0<\hat{x}=\sqrt{\frac{\sqrt{1+\chi_{2}}}{\sqrt{1+\chi_{1}}+\sqrt{1+\chi_{2}}}}<1, \hat{x} \in(0,1) \subseteq[-1,1] .
$$

Then

$$
\begin{aligned}
M_{2}(\hat{x}) & =\left(1+\chi_{1} \hat{x}^{2}\right)+\left(\chi_{2}+\chi_{3} \hat{x}^{2}\right)\left(1-\hat{x}^{2}\right)=1+\chi_{1} \frac{\sqrt{1+\chi_{2}}}{\sqrt{1+\chi_{1}}+\sqrt{1+\chi_{2}}} \\
& +\left(\chi_{2}+\chi_{3} \frac{\sqrt{1+\chi_{2}}}{\sqrt{1+\chi_{1}}+\sqrt{1+\chi_{2}}}\right)\left(1-\frac{\sqrt{1+\chi_{2}}}{\sqrt{1+\chi_{1}}+\sqrt{1+\chi_{2}}}\right),
\end{aligned}
$$

and after some manipulations we have

$$
M_{2}(x)=\frac{\sqrt{1+\chi_{1}} \sqrt{1+\chi_{2}}\left[\left(\sqrt{1+\chi_{1}}+\sqrt{1+\chi_{2}}\right)^{2}+\chi_{3}\right]}{\left(\sqrt{1+\chi_{1}}+\sqrt{1+\chi_{2}}\right)^{2}} \leq 0 .
$$

Thus according to Theorem 1 it is again in contradiction with the assumptions of the theorem.

Now we have to prove the second part of the theorem, namely: if the conditions $i$ ), ii), and $i i i)$ are satisfied then expressions $M_{i}, i=1,2,3$ are nonzero for each $x \in[-1,1]$.

a) Suppose that the conditions $i$ )-iii) are satisfied and, at the same time, there exists a point $\tilde{x}$ from the interval $[-1,1]$ such that

$$
M_{3}\left(\tilde{x}, \chi_{1}\right)=1+\chi_{1} \tilde{x}^{2}=0 .
$$

Because $M_{3}\left(0, \chi_{1}\right)=1$ then $\tilde{x} \neq 0$ and

$$
M_{3}\left(\tilde{x}, \chi_{1}\right)=1+\chi_{1} \tilde{x}^{2}=0 \stackrel{(\tilde{x} \neq 0)}{\Rightarrow} \chi_{1}=-\frac{1}{\tilde{x}^{2}} \Rightarrow \chi_{1} \leq-1,
$$

which is in contradiction with assumption $i)$. Thus, $M_{3}(x)>0$ for all $x \in[-1,1]$. 
b) As in the previous case, suppose that conditions $i$ )-iii) are satisfied and, at the same time, suppose the existence of $\tilde{x} \in[-1,1]$ such that $M_{2}\left(\tilde{x}, \chi_{1}, \chi_{2}, \chi_{3}\right)=0$. But

$$
M_{2}\left(0, \chi_{1}, \chi_{2}, \chi_{3}\right)=1+\chi_{2}>0, \quad M_{2}\left( \pm 1, \chi_{1}, \chi_{2}, \chi_{3}\right)=1+\chi_{1}>0 .
$$

Thus, if $M_{2}\left(\tilde{x}, \chi_{1}, \chi_{2}, \chi_{3}\right)=0$ then $\tilde{x} \neq 0 \wedge \tilde{x} \neq \pm 1$. As a result $\tilde{x}^{2} \in(0,1)$. Then we have

$$
\begin{aligned}
M_{2}\left(\tilde{x}, \chi_{1}, \chi_{2}, \chi_{3}\right) & =1+\chi_{1} \tilde{x}^{2}+\left(\chi_{2}+\chi_{3} \tilde{x}^{2}\right)\left(1-\tilde{x}^{2}\right) \\
& =\tilde{x}^{2}\left(1-\tilde{x}^{2}\right)\left[\frac{1+\chi_{1}}{1-\tilde{x}^{2}}+\frac{1+\chi_{2}}{\tilde{x}^{2}}+\chi_{3}\right]=0 .
\end{aligned}
$$

Because $\tilde{x} \neq 0 \wedge \tilde{x} \neq \pm 1$ then

$$
\frac{1+\chi_{1}}{1-\tilde{x}^{2}}+\frac{1+\chi_{2}}{\tilde{x}^{2}}+\chi_{3}=0
$$

which is equivalent to

$$
\chi_{3}=-\frac{1+\chi_{1}}{1-\tilde{x}^{2}}-\frac{1+\chi_{2}}{\tilde{x}^{2}} .
$$

Further, the maximum of function $f(t)=-\frac{1+\chi_{1}}{1-t}-\frac{1+\chi_{2}}{t}$ within the interval $t \in(0,1)$ is obtained at the point $t^{\star}=\frac{\sqrt{1+\chi_{2}}}{\sqrt{1+\chi_{1}}+\sqrt{1+\chi_{2}}}$ and its value is

$$
f\left(t^{\star}\right)=-\left(\sqrt{1+\chi_{1}}+\sqrt{1+\chi_{2}}\right)^{2} .
$$

Therefore $\chi_{3} \leq-\left(\sqrt{1+\chi_{1}}+\sqrt{1+\chi_{2}}\right)^{2}$, which is in contradiction with assumption $i i i)$. As a result $M_{2}(x)>0$ for every $x$ from the interval $[-1,1]$.

c) It is evident that

$$
M_{1}\left(x, \chi_{1}, \chi_{2}, \chi_{3}\right)=M_{2}\left(x, \chi_{1}, \chi_{2}, \chi_{3}\right)+M_{3}\left(x, \chi_{1}\right)
$$

and according to $a$ ) and $b$ ) one obtains

$$
M_{1}\left(x, \chi_{1}, \chi_{2}, \chi_{3}\right)=M_{2}\left(x, \chi_{1}, \chi_{2}, \chi_{3}\right)+M_{3}\left(x, \chi_{1}\right)>0
$$

for all $x \in[-1,1]$. Thus, the theorem is proved.

\section{References}

[1] L. Ts. Adzhemyan, N. V. Antonov and A. N. Vasil'ev. The problem of infrared divergences and renormalization group in theory of developed turbulence. $Z h$. Eks. Teor. Fiz., 95(4), 1272-1288, 1989. 
[2] L. Ts. Adzhemyan, N. V. Antonov and A. N. Vasiliev. The Field Theoretic Renormalization Group in Fully Developed Turbulence. Gordon \& Breach, London, 1999.

[3] L. Ts. Adzhemyan, M. Hnatich, D. Horvath and M. Stehlik. Infrared properties of an anisotropically driven MHD turbulence. Int. J. Mod. Phys. B, 9(26), 3401-3420, 1995.

[4] L. Ts. Adzhemyan, A. N. Vasil'ev and Yu. M. Pis'mak. Renormalization-group approach in the theory of turbulence: the dimensions of composite operators. Theor. Math. Phys., 57(1), 1131-1141, 1983.

[5] R. Bausch, H. K. Janssen and H. Wagner. Renormalization field theory of critical dynamics. Z. Physik B, 24(1), 113-127, 1976.

[6] J. Busa, M. Hnatic, E. Jurcisinova, M. Jurcisin and M. Stehlik. Influence of anisotropy on the scaling regimes in fully developed turbulence. Acta Phys. Slov., 52(6), 547-552, 2002.

[7] J. Busa, M. Hnatich, J. Honkonen and D. Horvath. Stability of kolmogorov scaling in anisotropically forced turbulence. Phys. Rev. E, 55(1), 381-394, 1997.

[8] C. DeDominicis and P. C. Martin. Energy spectra of certain randomly-stirred fluid. Phys. Rev. A, 19(1), 419-422, 1979.

[9] I. S. Gradshtejn and I. M. Ryzhik. Tables of Integrals, Series and Products. Academic, New York, 1965.

[10] E. A. Hayryan, J. Buša, E. E. Donets, I. Pokorný and O. I. Streltsova. Numerical studies of perturbed static solutions decay in the coupled system of YangMills-Dilaton equations with use of MPI technology. Journal of Mathematical Modeling (in Russian), 17(6), 103-121, 2005.

[11] E. A. Hayryan, E. Jurcisinova, M. Jurcisin, I. Pokorny and M. Stehlik. Stability of kolmogorov scaling in the theory of anisotropically driven developed turbulence. Communication of JINR E17-2005-208, 2005.

[12] M. Hnatic, E. Jonyova, M. Jurcisin and M. Stehlik. Stability of scaling regimes in $d \geq 2$ developed turbulence with weak anisotropy. Phys. Rev. E, 64(1), $016312,2001$.

[13] T. L. Kim and A. V. Serd'ukov. Quantum-field renormalization group in theory of developed turbulence: Inclusion of anisotropy and passive admixtur. Teor. Mat. Fiz., 105(2), 412-422, 1995.

[14] A. N. Kolmogorov. The local structure of turbulence in an incompressible fluid with very large reynolds numbers. Dokl. Akad. Nauk SSSR, 30(2), 301-305, 1941.

[15] P. C. Martin, E. D. Siggia and H. A. Rose. Statistical dynamics of classical systems. Phys. Rev. A, 8(1), 423-437, 1973.

[16] A. S. Monin and A. M. Yaglom. Statistical Fluid Mechanics. MIT Press, Cambridge, MA, 1975. Vol. 2

[17] A. M. Obukhov. On the distribution of energy in the spectrum of turbulent flow. Dokl. Akad. Nauk SSSR, 32(2), 22-24, 1941.

[18] R. Rubinstein and J. M. Barton. Infrared properties of an anisotropically stirred fluid. Phys. Fluids., 30(10), 2987-2992, 1987.

[19] A. N. Vasil'ev. The Field Theoretic Renormalization Group in Critical Behavior Theory and Stochastic Dynamics. Sankt Peterburg, 1998. in Russian, [English translation: Chapman \& Hall/CRC, Boca Roaton, FL, 2004]

[20] V. V. Voevodin and Vl. V. Voevodin. Parallel Computations. St.Peterburg, 2002. (in Russian)

[21] H. W. Wyld. Formulation of the theory of turbulence in an incompressible fluid. Ann. Phys., 14(1), 141-165, 1961. 